\title{
In vitro cell and tissue models for studying host-microbe interactions: a review
}

\author{
Miriam Bermudez-Brito, Julio Plaza-Díaz, Luis Fontana, Sergio Muñoz-Quezada and Angel Gil* \\ Department of Biochemistry \& Molecular Biology II, School of Pharmacy and Institute of Nutrition \& Food Technology \\ "José Mataix", Biomedical Research Centre, University of Granada, Granada, Spain
}

\begin{abstract}
Ideally, cell models should resemble the in vivo conditions; however, in most in vitro experimental models, epithelial cells are cultivated as monolayers, in which the establishment of functional epithelial features is not achieved. To overcome this problem, co-culture experiments with probiotics, dendritic cells and intestinal epithelial cells and three-dimensional models attempt to reconcile the complex and dynamic interactions that exist in vivo between the intestinal epithelium and bacteria on the luminal side and between the epithelium and the underlying immune system on the basolateral side. Additional models include tissue explants, bioreactors and organoids. The present review details the in vitro models used to study host-microbe interactions and explores the new tools that may help in understanding the molecular mechanisms of these interactions.
\end{abstract}

Key words: Probiotics: Cell models: Tissue explants: Bioreactors: Organoids

Over the course of evolution, lactobacilli, other lactic acid bacteria (LAB) and bifidobacteria have been abundant colonisers of the human small intestinal mucosa and coexist in mutualistic relationships with the host. Some members of these groups exert additional probiotic properties that provide health benefits to the host via the regulation of immune system and other physiological functions ${ }^{(1,2)}$.

The immune system can be divided into two systems: innate and adaptive. The adaptive immune response depends on B- and T-lymphocytes, which are specific for particular antigens. By contrast, the innate immune system responds to common structures, called pathogen-associated molecular patterns, which are shared by the vast majority of pathogens. The primary response to pathogens is triggered by the pattern recognition receptors that bind pathogen-associated molecular patterns; pattern recognition receptors comprise Toll-like receptors (TLR), nucleotide-binding oligomerisation domains, adhesion molecules and lectins ${ }^{(3)}$.

The use of probiotics is considered to be a potentially important strategy for modulating infectious and inflammatory responses in the gastrointestinal tract of the host. The effect of these probiotics is diverse and includes the modulation of the gut immune system through the interaction with gut epithelial cells and immune cells. These interactions primarily involve gut-associated dendritic cells (DC), which have the capability to respond to microbial signals through TLR signalling ${ }^{(4-6)}$.

For a micro-organism to qualify as a probiotic, it is essential to scientifically demonstrate that it is beneficial to the health of the host. Before testing probiotics in human subjects, a sine qua non condition is to conduct studies in cell and animal models. In vitro and animal studies may provide valuable information, such as the mechanism through which a probiotic acts, but these types of studies alone are not proof of the benefit of a putative probiotic to human health.

Intestinal epithelial cells (IEC) are a barrier between the intestinal lumen and host connective tissue. However, recent studies have demonstrated that IEC are involved in the immunological process of the discrimination between pathogenic and commensal bacteria. IEC also secrete a broad range of antimicrobial peptides, including defensins, cathelicidins and calprotectins. The IEC interact with subepithelial professional antigen-presenting cells that can sample antigens and micro-organisms and are mostly populations of DC and macrophage-associated lymphoid tissues. These subepithelial cells are able to polarise naïve $\mathrm{T}$ cells and produce an immunotolerance or an inflammatory response. The interaction of IEC, DC and macrophages with commensal or pathogenic bacteria stimulates the differential secretion of cytokines. Thymic stromal lymphopoietin and IL-10 are secreted by IEC

Abbreviations: 3D, three-dimensional; DC, dendritic cells; DCSIGN, dendritic cells-specific intercellular adhesion molecule 3-grabbing non-integrin; IEC, intestinal epithelial cells; IFN, interferon; LAB, lactic acid bacteria; MDC, myeloid dendritic cells; PSC, pluripotent stem cells; Th, T helper; TLR, Toll-like receptors; $\mathrm{T}_{\text {reg, }}$, regulatory $\mathrm{T}$ cells.

*Corresponding author: Professor Á. Gil, fax +34958 819132, email agil@ugr.es 
in the presence of commensal or probiotic bacteria, whereas the pro-inflammatory cytokines IL- 8 and TNF- $\alpha$ are secreted when pathogenic bacteria are present ${ }^{(7)}$. DC can sample antigens directly from the intestinal lumen by forming tightjunction-like structures with $\operatorname{IEC}^{(8)}$; alternatively, DC can be stimulated by the cytokines secreted from IEC. An environment containing cytokines secreted by IEC and DC stimulates immature DC and macrophages to produce a further increase in the level of cytokines. The subepithelial naïve $\mathrm{T}$ cells are stimulated for immunotolerance or in response to a profile of inflammatory cytokines and promote the generation of Th1, Th2, Th17 or regulatory $\mathrm{T}$ cells $\left(\mathrm{T}_{\text {reg }}\right)$. Because this complex net of secreted cells and cytokines is not easy to mimic in vitro, different models have been developed that principally involve IEC and DC generated from monocytes or segments of intestine (rat, human $)^{(9-11)}$ and $\mathrm{T}$ cells isolated from peripheral blood mononuclear cells ${ }^{(11)}$. Such models may include only one type of cell, a co-culture of various cell types or, in an effort to mimic the intestinal tissue, a culture of explants from the intestines ${ }^{(12)}$. Often, the models determine the different cytokines secreted or distinguish between different

Intestinal epithelial cells

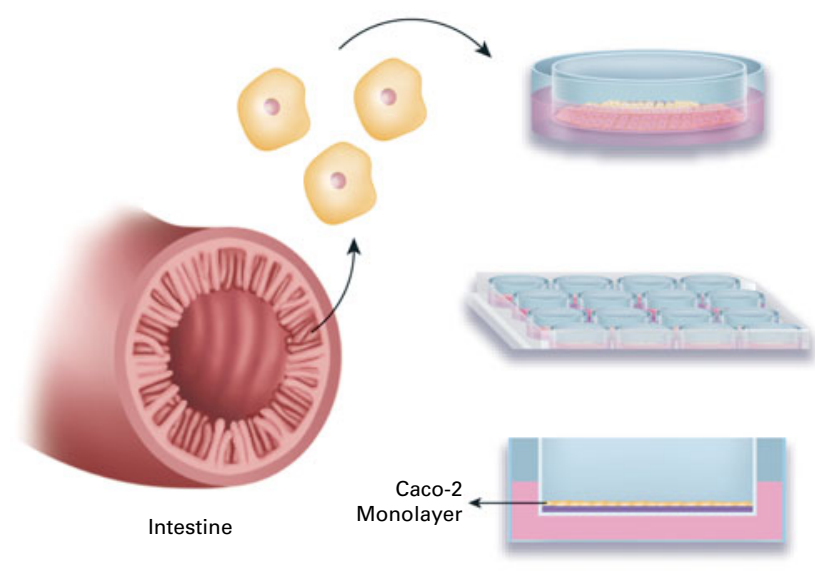

Intestinal explant culture

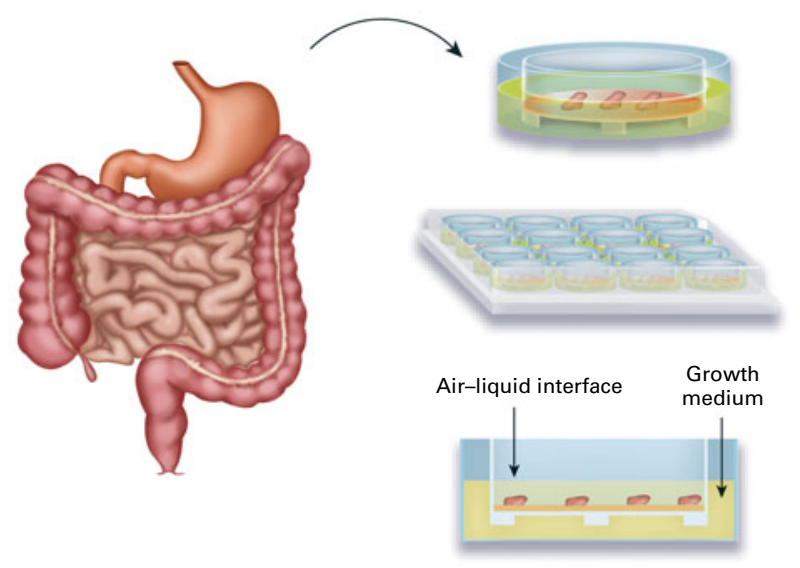

subsets of DC or $\mathrm{T}$ cells using cell surface phenotypes or transcription factors that are specific for each cell type. Here, we review the cell and tissue models that are currently used or could potentially be used to ascertain the mechanism through which probiotics act (Fig. 1).

\section{In vitro models}

Although human clinical trials are the definitive tool for establishing probiotic functionality, the use of in vitro models is necessary to select the most promising strains for these trials. Several in vitro studies evaluate the adhesion ability of potential probiotic bacteria and their interactions with pathogens at the intestinal epithelial interface ${ }^{(13-15)}$. The main goals of these studies are to understand the immunomodulatory effects of different bacterial strains on in vitro cell models and to evaluate whether the strain-dependent characteristics of commensal bacteria make them appropriate strains for the prevention and treatment of diseases.

A wide variety of cells are used as in vitro models for probiotic evaluation. Available models include both normal and

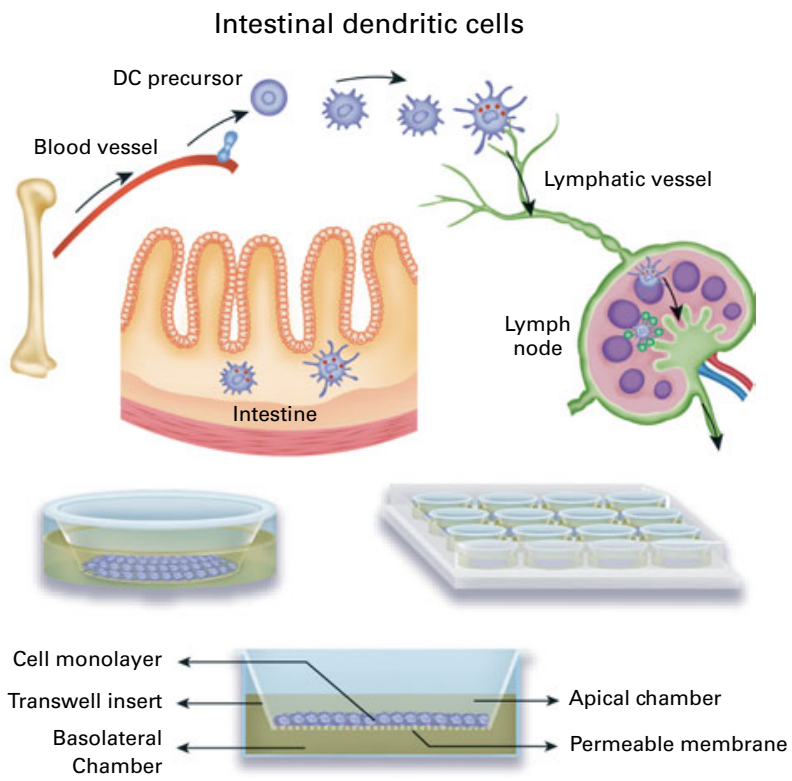

Intestinal organoid culture
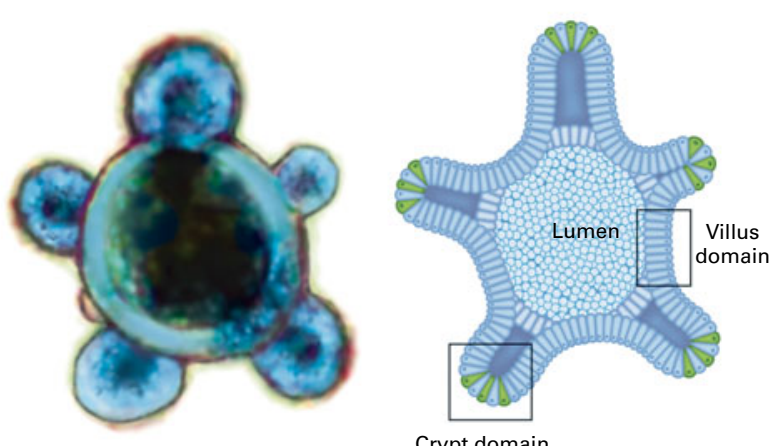

Crypt domain

Fig. 1. Available in vitro models for studying host-microbe interactions and the mechanisms of action of probiotics. DC, dendritic cells. 
carcinogenic cells of different origins (intestine and blood), species (human, rat, pig, calf, goat, sheep and chicken) and types (epithelial and monocyte/macrophage).

\section{Intestinal epithelial cells}

Three of the most widely used commercially available human cell lines are Caco-2, T84 and HT-29 ${ }^{(16,17)}$, all of which were isolated from colon adenocarcinomas, express the features of enterocytes and are useful for attachment and mechanistic studies. In the differentiated state, these cell lines mimic the typical characteristics of the human small intestinal epithelium, including a well-developed brush border with such associated enzymes as alkaline phosphatase and sucrose isomaltase ${ }^{(18)}$. The HT29-MTX is a cell line obtained from HT29 cells adapted to methotrexate ${ }^{(19)}$, which differentiate into goblet cells and secrete mucin, although of gastric immunoreactivity ${ }^{(20,21)}$. Nevertheless, these three cell models are different from the small intestine in several aspects, and their phenotypes are dependent on the duration of the culture period ${ }^{(22,23)}$. Enterocytes and goblet cells represent the two major cell phenotypes in the intestinal epithelium, and IEC- 6 and IEC-18 are the most widely used among the rodent cell lines ${ }^{(24,25)}$. Both are commercially available and are derived from normal (noncarcinogenic) rat small intestine. Other available cell lines have been extensively reviewed by Cencič \& Langerholc ${ }^{(26)}$.

The models of IEC are focused on studying the receptors of the innate immune system (TLR and nucleotide oligomerization domain (NOD)) and the pathways that result in the secretion of cytokines. Ma et al. ${ }^{(27)}$ showed that live Lactobacillus reuteri cells were able to reduce $\mathrm{TNF}-\alpha$-induced IL (IL-8) levels in Caco-2 cells. In addition, Vizoso Pinto et al. ${ }^{(28)}$ showed that TLR9 and TLR2 were up-regulated when HT29 cells were incubated with lactobacilli but not when incubated only with Salmonella typhimurium. Using polarised HT29 and T84 cell monolayers, Ghadimi et al. ${ }^{(29)}$ demonstrated that apically applied DNA from Lactobacillus rhamnosus GG (a human commensal and probiotic bacteria) attenuated TNF- $\alpha$-enhanced NF- $\kappa \mathrm{B}$ activity by reducing the degradation of the inhibitor subunit $\alpha$ of NF-кB (IкB $\alpha)$ and $\mathrm{p} 38$ subunit mitogen-activated protein kinase phosphorylation. In vitro studies have suggested that through the secretion of such immunoregulatory molecules as IL-8, TNF- $\alpha$, TSLP, transforming growth factor- $\beta$ and $\mathrm{PGE}_{2}$, IEC limit pro-inflammatory cytokine production in DC. Thus, the secretion of immunoregulatory molecules by IEC is important for the maintenance of intestinal immune homeostasis ${ }^{(30,31)}$.

\section{Dendritic cells}

DC comprise a complex, heterogeneous group of multifunctional antigen-presenting cells that comprise a critical arm of the immune system ${ }^{(32-35)}$. DC differentiate into at least four lines: Langerhans cells, myeloid DC (MDC), lymphoid DC and plasmacytoid $\mathrm{DC}^{(36)}$. These cells play critical roles in the orchestration of the adaptive immune response by inducing both tolerance and immunity ${ }^{(37-39)}$. The present paradigm is that this dual role results from the division of the total
DC population into a network of DC subsets having distinct functions $^{(36,40)}$.

Immature DC reside in peripheral tissues, such as the gut mucosa, where they sense the microenvironment via pattern recognition receptors, including TLR and C-type lectin receptors, which recognise pathogen-associated molecular patterns ${ }^{(41)}$. Immature DC also release chemokines and cytokines to amplify the immune response ${ }^{(35)}$. Therefore, the regulatory role of DC is of particular importance at such mucosal surfaces as the intestine, where the immune system exists in intimate association with commensal bacteria, including $\mathrm{LAB}^{(42)}$. Probiotics exert differential stimulatory effects on DC in vitro, giving rise to varying production levels of different cytokines and, accordingly, different effector functions $^{(43-45)}$.

The response of the immune system to probiotics remains controversial. Some strains modulate the cytokine production by DC in vitro and induce a regulatory response, whereas others induce a pro-inflammatory response ${ }^{(46)}$. These straindependent effects are thought to be linked to specific interactions between bacteria and pattern recognition receptors.

Braat et al. ${ }^{(47)}$ proposes that L. rhamnosus modulates DC function to induce a novel form of T-cell hyporesponsiveness, a mechanism that might be an explanation for the observed beneficial effects of probiotic treatment in clinical diseases.

The analysis of immature bone marrow-derived DC showed that all strains up-regulated the surface expression of B7-2 (CD86), which is indicative of DC maturation. However, the different strains up-regulated CD86 with varying intensities. No strain induced appreciable levels of IL-10 or IL-12 in immature bone marrow-derived DC, whereas TNF- $\alpha$ expression was elicited by Lactobacillus paracasei and Lactobacillus fermentum $^{(48)}$ in particular.

Although efficiently taken up by DC in vitro, selected LAB strains induced only a partial maturation of $\mathrm{DC}^{(43,49)}$. The transfer of probiotic-treated DC conferred protection against 2,4,6-trinitrobenzenesulfonic acid solution-induced colitis, and the preventive effect required Myeloid differentiation primary (MyD88)-, TLR2- and NOD2-dependent signalling and also the induction of $\mathrm{CD} 4+\mathrm{CD} 25+$ regulatory cells in an IL-10-independent pathway ${ }^{(49)}$.

Mohamadzadeh et al. ${ }^{(50)}$ investigated three species of Lactobacillus and found that they modulated the phenotype and function of human MDC. Lactobacillus-exposed MDC up-regulated human leukocyte antigen-DR (HLA-DR), CD83, CD40, CD80 and CD86 and secreted high levels of IL-12 and IL-18 but not IL-10. IL-12 was sustained in the MDC exposed to all three of the Lactobacillus species in the presence of lipopolysaccharide from Escherichia coli, whereas lipopolysaccharide-induced IL-10 was greatly inhibited. The MDC activated with lactobacilli clearly skewed the $\mathrm{CD} 4^{+}$and $\mathrm{CD}^{+}$ $\mathrm{T}$ cells to $\mathrm{T}$ helper (Th) 1 and $\mathrm{T}$ cell 1 (Tc1) polarisation, as evidenced by the secretion of interferon (IFN) $-\gamma$ but not IL- 4 or IL-13.

L. reuteri and Lactobacillus casei, but not Lactobacillus plantarum, prime monocyte-derived DC to drive the development of $\mathrm{T}_{\text {reg }}$ cells. The $\mathrm{T}_{\text {reg }}$ cells then produce increased levels of IL-10 and are capable of inhibiting the proliferation of 
bystander $\mathrm{T}$ cells in an IL-10-dependent fashion. Strikingly, both $L$. reuteri and $L$. casei, but not $L$. plantarum, bind the C-type lectin DC-specific intercellular adhesion molecule 3-grabbing non-integrin (DCSIGN). Blocking antibodies against DCSIGN inhibited the induction of the $\mathrm{T}_{\text {reg }}$ cells by these probiotic bacteria, stressing that binding of DCSIGN can actively prime DC to induce $\mathrm{T}_{\text {reg }}$ cells ${ }^{(11)}$.

All bifidobacteria and certain lactobacilli strains are low IL-12 and TNF- $\alpha$ inducers, and the IL-10 and IL- 6 levels showed less variation than and no correlation with IL-12 and TNF- $\alpha$. The DC matured by strong IL-12-inducing strains also produced high levels of IFN- $\beta$. When combining two strains, the low IL-12 inducers inhibited both this IFN- $\beta$ production and the IL-12 and Th1-skewing chemokines. Weiss et al $^{(51)}$ demonstrate that lactobacilli can be divided into two groups of bacteria that have contrasting effects; conversely, all bifidobacteria exhibit uniform effects.

In conclusion, LAB potently initiate 'interactions' via DC maturation. Hence, these LAB strains may represent useful tools for modulating the cytokine balance and promoting potent type- 1 immune responses or preventing the immune deregulation associated with specific T-cell polarisation ${ }^{(45)}$.

\section{Macrophages}

Lactobacilli have been shown to activate monocytes and macrophages, which play pivotal roles in antigen processing and presentation, the activation of antigen-specific immunity and the stimulation of IgA immunity. In particular, these cells are essential in the deviation of the immune response to the so-called type 1 response with cytotoxic effector cells or towards the type 2 response that is characterised by antibody production. The type 2 response is related to the secretion of IL-4, IL-5, IL-9 and IL-13, which promote the induction of $\operatorname{IgE}$ and allergic responses. By incubating bacterial suspensions with THP-1 macrophage-like cells, Drago et al. ${ }^{(52)}$ analysed four strains of Lactobacillus salivarius for their ability to modulate the release of pro- and anti-inflammatory cytokines. LDR0723 and CRL1528 led to a sustained increase in the production of IL-12 and IFN- $\gamma$ and a decrease in the release of IL- 4 and IL-5; by contrast, BNL1059 and RGS1746 favoured a Th2 response, leading to a decrease in the Th1:Th2 ratio with respect to unstimulated cells.

Ivec et al. ${ }^{(53)}$ showed that probiotic bacteria, either from Lactobacillus sp. or bifidobacteria, have the ability to decrease viral infection by establishing the antiviral state in macrophages through the production of $\mathrm{NO}$ and inflammatory cytokines, such as IL-6 and IFN- $\gamma$.

\section{Three-dimensional cell models and probiotics}

Much of the present knowledge about how microbial pathogens cause infection is based on studying experimental infections of standard cell monolayers grown as flat twodimensional cultures on impermeable glass or plastic surfaces. Whereas these models continue to contribute to the present understanding of infectious diseases, they are greatly limited because they are unable to model the complexity of intact three-dimensional (3D) tissue ${ }^{(54,55)}$. In addition, cells grown as standard two-dimensional monolayers are also unable to respond to chemical and molecular gradients in three dimensions (at the apical, basal and lateral cell surfaces), resulting in many departures from the in vivo behaviour ${ }^{(56)}$

There are a variety of methods that have been used to enhance the differentiation of cultured cells, including permeable inserts, transplanted human cells grown as xenografts in animals and explanted human biopsies ${ }^{(57)}$. Together, with animal experiments, organotypical cell cultures are important models for analysing the cellular interactions of the mucosal epithelium and pathogenic mechanisms in the gastrointestinal tract $^{(58)}$. Although these advanced in vitro models have provided important insights into microbial pathogenesis, they suffer from several limitations, including short lifetimes, labour-intensive design, experimental variability, availability and limited numbers of cells ${ }^{(57,59)}$.

\section{Three-dimensional cell cultures and probiotics}

Epithelial cells cultured in a 3D matrix self-assemble into polarised monolayers that separate central apical lumens from a basal environment containing extracellular matrix; therefore, these 3D epithelial culture systems allow key events in the life cycle of IEC, such as proliferation, differentiation, apoptosis and migration, to be controlled in concert by organising principles that are determined by the spatial context of the cells. 3D culture systems mimic essential aspects of the in vivo organisation of epithelial cells of various origins. Compared with cell monolayers, the 3D culture of human intestinal cell lines (small intestine and colon) enhanced many characteristics associated with fully differentiated functional intestinal epithelia in vivo, including a distinct apical and basolateral polarity, the increased expression and better organisation of the tight junctions, extracellular matrix and brush border proteins and the highly localised expression of mucins. All of these important physiological features of in vivo intestinal epithelium were either absent or not expressed or distributed at physiologically relevant levels in monolayer cultures of the same cells ${ }^{(60)}$.

Recently, Mappley et al. ${ }^{(61)}$ reported a study using a 3D culture model and probiotics. In in vitro adherence and invasion assays using HT29-16E 3D cells, the adherence and invasion of Brachyspira pilosicoli B2904 into epithelial cells were significantly reduced by the presence of the cell-free supernatants of two Lactobacillus strains, L. reuteri LM1 and L. salivarius LM2.

\section{Tissue explants and probiotics}

A limited number of studies used explants and probiotics, and all of these studies focused on intestinal diseases, particularly Crohn's disease.

Using an organ-culture model with intestinal mucosa explants and selected bacterial strains, Carol et al. ${ }^{(62)}$ reported a decrease of activated T lymphocytes and TNF- $\alpha$ secretion by the inflamed mucosa of patients with Crohn's disease. By favouring the apoptosis of T lymphocytes, L. casei may restore 
immune homeostasis in the inflamed ileal mucosa of these patients. In addition, Carol et al. ${ }^{(62)}$ also demonstrated that some lactobacilli, such as L. casei DN-11 401 and L. bulgaricus LB10, may down-regulate inflammatory responses when exposed to inflamed mucosa in organ culture ${ }^{(63,64)}$. These authors conclude that probiotics interact with immunocompetent cells through the mucosal interface and locally modulate the production of pro-inflammatory cytokines. It is important to note that, although this model is useful for studying whole tissue responses in Crohn's disease, it does not permit the investigation of signals between mucosal cells because mucosa explants include a great variety of cells in their natural disposition for cell-to-cell communication.

More recently, Mencarelli et al. ${ }^{(65)}$ cultured abdominal fat explants from five Crohn's disease patients and five patients with colon cancer (as controls) using VSL\#3 (VSL Pharmaceuticals, Fort Lauderdale FL) medium and found that the exposure of these tissues to the VSL $\# 3$ conditioned medium abrogates leptin release. Thus, probiotics seem to correct inflammation-driven metabolic dysfunction.

Lastly, another group treated mouse colon epithelial cells and cultured colon explants with purified L. rhamnosus GG proteins in the absence or presence of TNF- $\alpha$. Two novel purified proteins p75 and p40 activated protein kinase B (Akt), inhibited cytokine-induced epithelial cell apoptosis and promoted cell growth in human and mouse colon epithelial cells and cultured mouse colon explants. Furthermore, TNF-induced colon epithelial damage was significantly reduced. These findings suggest that probiotic bacterial components may be useful for preventing cytokine-mediated gastrointestinal diseases ${ }^{(66)}$.

\section{Future models}

3D culture advanced in vitro models have provided important insights into microbial pathogenesis. However, they have several limitations, including short lifetimes, labour-intensive design, experimental variability, availability and limited numbers of cells. The development of novel relevant in vitro models of human intestinal epithelium, such as bioreactors and organoids, provides a viable starting point for future efforts aimed at bioengineering human intestine. 3D culture in bioreactors represents an easy, reproducible and highthroughput platform that provides a large number of differentiated cells. Another promising line is human pluripotent stem cells (PSC) that offer a unique and promising means to generate intestinal tissue, resulting in 3D intestinal 'organoids' formed villus-like structures and crypt-like proliferative zones. This intestinal tissue is functional, as it can secrete mucins into luminal structures.

\section{Bioreactors}

Tissue engineering represents a biology-driven approach by which bioartificial tissues are engineered through the combination of material technology and biotechnology. Bioreactors constitute and maintain physiological tissue conditions at desired levels, enhance mass transport rates and expose cultured cells to specific stimuli. It has been shown that bioreactor technologies providing appropriate biochemical and physiological regulatory signals guide cell and tissue differentiation and influence the tissue-specific function of bioartificial 3D tissues.

BioVaSc. BioVaSc is generated from a decellularised porcine small bowel segment with preserved tubular structures of the capillary network within the collagen matrix. BioVaSc is a prerequisite technique for the generation of bioartificial tissues endowed with a functional artificial vascular network. The technology has been performed in artificial human liver, intestine, trachea and skin models. These various human tissue models are a new technology that is an alternative to animal experiments for pharmacokinetic (drug penetration, distribution and metabolisation) and pharmacodynamic studies ${ }^{(67)}$, and also to study probiotic interactions with the host.

Rotating-wall vessel bioreactor. Another alternative model utilises rotating-wall vessel technology to engineer biologically meaningful 3D models of human large intestinal epithelia and can be used in conjunction with the established models. Many reports have described the fact that cells cultured in an rotating-wall vessel bioreactor can assume physiologically relevant phenotypes that have not been possible with other models. In addition, 3D culture in rotating-wall vessel bioreactors represents an easy, reproducible and high-throughput platform that provides a large number of differentiated cells.

Optimally, the design of cell culture models should mimic both the 3D organisation and differentiated function of an organ, while allowing for experimental analysis in a highthroughput platform. Originally designed by National Aeronautics and Space Administration (NASA) engineers, the rotating-wall vessel technology is an optimised suspension culture design for growing 3D cells that maintain many of the specialised features of in vivo tissues ${ }^{(68,69)}$.

To date, several works have shown the use of $3 \mathrm{D}$ cell culture systems in infection studies with the following pathogens: S. typhimurium (small intestine and colon models) ${ }^{(59,70)}$, Pseudomonas aeruginosa (lung model) ${ }^{(71,72)}$, human cytomegalovirus (placental model) ${ }^{(73)}$ and Hepatitis $\mathrm{C}$ virus (hepatocyte model $)^{(74)}$. Additional studies with other $3 \mathrm{D}$ models and infectious agents are ongoing and include bacteria, viruses and parasites that are difficult or impossible to culture using conventional methods.

\section{Organoids}

Intestinal resection and malformations in adult and paediatric patients result in devastating consequences. Unfortunately, allogeneic transplantation of intestinal tissue into patients has not been met with the same measure of success as the transplantation of other organs. Attempts to engineer intestinal tissue in vitro include the disaggregation of adult rat intestine into subunits called organoids, harvesting native adult stem cells from mouse intestine and spontaneous generation of intestinal tissue from embryoid bodies ${ }^{(75)}$. Recently, by utilising principles gained from the study of developmental biology, human PSC have been demonstrated to be capable of directed differentiation into intestinal tissue in vitro ${ }^{(76)}$. 
PSC offer a unique and promising means to generate intestinal tissue for the purposes of modelling intestinal disease, understanding embryonic development and providing a source of material for therapeutic transplantation ${ }^{(75)}$. For example, human PSC have been differentiated into monolayer cultures of liver hepatocytes and pancreatic endocrine cells ${ }^{(77-80)}$ that have therapeutic efficacy in animal models of liver disease ${ }^{(80-82)}$ and diabetes ${ }^{(83)}$, respectively.

Several authors have differentiated PSC from mice and human subjects into intestinal tissue. The resulting 3D intestinal 'organoids' consisted of a polarised, columnar epithelia that were patterned into villus-like structures and crypt-like proliferative zones that expressed intestinal stem cell markers. The epithelia contained the normal number of Lgr5-positive stem cells, Paneth cells and transit-amplifying cells in the crypt domain and the three differentiated cell lineages (enterocytes, goblet and enteroendocrine cells) of the villus domain $^{(84)}$. This intestinal tissue is functional, as it can secrete mucins into luminal structures ${ }^{(76,85)}$. Furthermore, as based on defined growth factors and Matrigel, this well-established culture system retains critical in vivo characteristics, such as lineage composition and self-renewal kinetics ${ }^{(86)}$.

However, despite offering such great potential, this system is not without its limitations. For example, the intestinal organoids lack several components of the intestine in vivo, such as the enteric nervous system and the vascular, lymphatic and immune systems. Additionally, whereas all of the major epithelial cell types are generated in proportions similar to those found in vivo, and there is evidence of crypt-like domains housing stem cells, the 3D architecture is not as regular as that seen in vivo, and the villus-like structures are variable from one organoid to the next ${ }^{(85)}$. Regardless of these drawbacks, this system has extraordinary experimental utility for understanding and modelling human intestinal development, homeostasis and disease. Moreover, this system provides a viable starting point for future efforts aimed at bioengineering human intestine.

Finally, Sato et $a l .^{(86)}$ developed a technology that can be used to study infected, inflammatory or neoplastic tissues from the human gastrointestinal tract. Encouraged by the establishment of murine small intestinal cultures, these researchers adapted that culture condition to mouse and human colonic epithelia. However, long-term adult human IEC culture has remained difficult. Although there have been some long-term culture models, these techniques and cell lines have not gained wide acceptance, possibly as a result of the inherent technical difficulties in extracting and maintaining viable cells. These tools might have applications in regenerative biology through ex vivo expansion of the intestinal epithelia.

\section{Conclusions}

In most in vitro experimental models, epithelial cells are cultivated as monolayers, in which the establishment of functional epithelial features is not achieved. Compared with cell monolayers, the 3D culture of human intestinal cell lines enhanced many characteristics associated with fully differentiated functional intestinal epithelia in vivo. However, despite providing important insights, a number of limitations have to be taken into account, including short lifetimes, labour-intensive design, experimental variability, availability and limited numbers of cells.

Considerable effort has been invested in the development of new tools to study host-microbe interactions. Recent studies based on the ability to generate human intestinal tissues may help in understanding the molecular mechanisms of host-microbe interactions. Bioreactors and organoids provide a viable starting point for future efforts aimed at bioengineering human intestine. However, to date, these new approaches have also limitations that must be considered. For example, in organoids, the 3D architecture is not as regular as that seen in vivo and the villus-like structures are variable from one organoid to the next.

\section{Acknowledgements}

The authors declare that they have no conflict of interest. Part of the research currently in progress in our laboratory is funded by the company Hero España, S.A. through the grants no. 3143 and 3545 managed by the Fundación General Empresa-Universidad de Granada. The author contributions were as follows: L. F. and M. B.-B. wrote the abstract; J. P.-D., S. M.-Q. and L. F. wrote the introduction and the section 'In vitro models'. M. B.-B. and A. G. wrote the sections 'Three dimensional models and probiotics', 'Future models' and 'Conclusions'. J. P.-D. and S. M.-Q. made the figure.

\section{References}

1. Konstantinov SR, Smidt H, de Vos WM, et al. (2008) S layer protein A of Lactobacillus acidophilus NCFM regulates immature dendritic cell and $\mathrm{T}$ cell functions. Proc Natl Acad Sci U S A 105, 19474-19479.

2. MacDonald TT \& Monteleone G (2005) Immunity, inflammation, and allergy in the gut. Science 307, 1920-1925.

3. McCole DF \& Barret KE (2007) Varied role of the gut epithelium in mucosal homeostasis. Curr Opin Gastroenterol 23, 647-654.

4. Pamer EG (2007) Immune responses to commensal and environmental microbes. Nat Immunol 11, 1173-1178.

5. Coombes JL \& Powrie F (2008) Dendritic cells in intestinal immune regulation. Nat Rev Immunol 8, 435-446.

6. Goriely S, Neurath MF \& Goldman M (2008) How microorganisms tip the balance between interleukin-12 family members. Nat Rev Immunol 8, 81-86.

7. Wallace TD, Bradley S, Buckley ND, et al. (2003) Interactions of lactic acid bacteria with human intestinal epithelial cells: effects on cytokine production. J Food Prot 66, 466-472.

8. Rescigno M, Urbano M, Valzasina B, et al. (2001) Dendritic cells express tight junction proteins and penetrate gut epithelial monolayers to sample bacteria. Nature Immunol 2, 361-367.

9. Uematsu S, Fujimoto K, Jang MH, et al. (2008) Regulation of humoral and cellular gut immunity by lamina propria dendritic cells expressing Toll-like receptor 5. Nat Immunol 9, 769-776.

10. Ng SC, Benjamin JL, McCarthy NE, et al. (2011) Relationship between human intestinal dendritic cells, gut microbiota, 
and disease activity in Crohn's disease. Inflamm Bowel Dis 17, 2027-2037.

11. Smits HH, Engering A, van der Kleij D, et al. (2005) Selective probiotic bacteria induce IL-10-producing regulatory $\mathrm{T}$ cells in vitro by modulating dendritic cell function through dendritic cell-specific intercellular adhesion molecule 3-grabbing nonintegrin. J Allergy Clin Immunol 115, 1260-1267.

12. Jarry A, Bossard C, Sarrabayrouse G, et al. (2011) Loss of interleukin-10 or transforming growth factor $\beta$ signaling in the human colon initiates a T-helper 1 response via distinct pathways. Gastroenterology 141, 1887-1896.

13. Sanz Y, Nadal I \& Sánchez E (2007) Probiotics as drugs against human gastrointestinal infections. Recent Pat Antiinfect Drug Discov 2, 148-156.

14. Izquierdo E, Medina M, Ennahar S, et al. (2008) Resistance to simulated gastrointestinal conditions and adhesion to mucus as probiotic criteria for Bifidobacterium longum strains. Curr Microbiol 56, 613-618.

15. Sánchez E, Nadal I, Donat E, et al. (2008) Reduced diversity and increased virulence-gene carriage in intestinal enterobacteria of coeliac children. BMC Gastroenterol 8, 50 .

16. Fogh J \& Trempe G (1975) New human tumor cell lines. In Human Tumor Cells In Vitro, pp. 115-140 [J Fogh, editor]. New York, NY: Plenum.

17. Fogh J, Fogh JM \& Orfeo T (1977) One hundred and twenty-seven cultured human tumor cell lines producing tumors in nude mice. $J$ Natl Cancer Inst 59, 221-226.

18. Lenaerts K, Bouwman FG, Lamers WH, et al. (2007) Comparative proteomic analysis of cell lines and scrapings of the human intestinal epithelium. BMC Genomics $\mathbf{8}, 91$.

19. Lesuffleur T, Barbat A, Dussaulx E, et al. (1990) Growth adaptation to methotrexate of HT-29 human colon carcinoma cells is associated with their ability to differentiate into columnar absorptive and mucus-secreting cells. Cancer Res 50, 6334-6343.

20. Lesuffleur T, Porchet N, Aubert JP, et al. (1993) Differential expression of the human mucin genes MUC1 to MUC5 in relation to growth and differentiation of different mucussecreting HT-29 cell subpopulations. J Cell Sci 106, 771-783.

21. Leteurtre E, Gouyer V, Rousseau K, et al. (2004) Differential mucin expression in colon carcinoma HT-29 clones with variable resistance to 5-fluorouracil and methotrexate. Biol Cell 96, 145-151.

22. Engle MJ, Goetz GS \& Alpers DH (1998) Caco-2 cells express a combination of colonocyte and enterocyte phenotypes. J Cell Physiol 174, 362-369.

23. Mehran M, Levy E, Bendayan M, et al. (1997) Lipid, apolipoprotein, and lipoprotein synthesis and secretion during cellular differentiation in Caco-2 cells. In Vitro Cell Dev Biol Anim 33, 118-128.

24. Quaroni A, Isselbacher KJ \& Ruoslahti E (1978) Fibronectin synthesis by epithelial crypt cells of rat small intestine. Proc Natl Acad Sci U S A 75, 5548-5552.

25. Quaroni A \& Isselbacher KJ (1981) Cytotoxic effects and metabolism of benzo[a]pyrene and 7,12-dimethylbenz[a]anthracene in duodenal and ileal epithelial cell cultures. $J$ Natl Cancer Inst 67, 1353-1362.

26. Cencič A \& Langerholc T (2010) Functional cell models of the gut and their applications in food microbiology - a review. Int J Food Microbiol 141, S4-S14.

27. Ma D, Forsythe P \& Bienenstock J (2004) Live Lactobacillus reuteri is essential for the inhibitory effect on tumor necrosis factor alpha-induced interleukin (IL)-8 expression. Infect Immunol 72, 5308-5314.

28. Vizoso Pinto MG, Rodriguez Gómez M, Seifert S, et al. (2009) Lactobacilli stimulate the innate immune response and modulate the TLR expression of HT29 intestinal epithelial cells in vitro. Int J Food Microbiol 31, 86-93.

29. Ghadimi D, De Vrese M, Heller KJ, et al. (2010) Effect of natural commensal-origin DNA on toll-like receptor 9 (TLR9) signalling cascade, chemokine IL-8 expression, and barrier integrity of polarized intestinal epithelial cells. Inflamm Bowel Dis 16, 410-427.

30. Wang J \& Xing F (2008) Human TSLP-educated DCs. Cell Mol Immunol 5, 99-106.

31. Dignass AU \& Podolsky DK (1993) Cytokine modulation of intestinal epithelial cell restitution: central role of transforming growth factor $\beta$. Gastroenterology 105, 1323-1332.

32. Steinman RM \& Banchereau J (2007) Taking dendritic cells into medicine. Nature 449, 419-426.

33. Banchereau J (2002) The long arm of the immune system. Sci Am 287, 52-59.

34. Banchereau J, Briere F, Caux C, et al. (2000) Immunobiology of dendritic cells. Annu Rev Immunol 18, 767-811.

35. Banchereau J \& Steinman RM (1998) Dendritic cells and the control of immunity. Nature 392, 245-252.

36. Wu L \& Liu YJ (2007) Development of dendritic-cell lineages. Immunity 26, 741-750.

37. Cools N, Ponsaerts P, Van Tendeloo VF, et al. (2007) Balancing between immunity and tolerance: an interplay between dendritic cells, regulatory $\mathrm{T}$ cells, and effector $\mathrm{T}$ cells. J Leukoc Biol 82, 1365-1374.

38. Quah BJ \& O'Neill HC (2005) Maturation of function in dendritic cells for tolerance and immunity. J Cell Mol Med 9, 643-654.

39. Steinman RM, Hawiger D \& Nussenzweig MC (2003) Tolerogenic dendritic cells. Annu Rev Immunol 21, $685-711$

40. Shortman K \& Naik SH (2007) Steady-state and inflammatory dendritic-cell development. Nat Rev Immunol 7, 19-30.

41. Sabatté J, Maggini J, Nahmod K, et al. (2007) Interplay of pathogens, cytokines and other stress signals in the regulation of dendritic cell function. Cytokine Growth Factor Rev 18, 5-17.

42. Stagg AJ, Hart AL, Knight SC, et al. (2004) Microbial-gut interactions in health and disease. Interactions between dendritic cells and bacteria in the regulation of intestinal immunity. Best Pract Res Clin Gastroenterol 18, 255-270.

43. Christensen HR, Frøkiær H \& Pestka JJ (2002) Lactobacilli differentially modulate expression of cytokines and maturation surface markers in murine dendritic cells. J Immunol 168, $171-178$

44. Zeuthen LH, Fink LN \& Frøkiær H (2008) Epithelial cells prime the immune response to an array of gut-derived commensals towards a tolerogenic phenotype through distinct actions of thymic stromal lymphopoietin and transforming growth factor- $\beta$. Immunology 123, 197-208.

45. Fink LN, Zeuthen LH, Ferlazzo G, et al. (2007) Human antigen-presenting cells respond differently to gut-derived probiotic bacteria but mediate similar strain-dependent NK and $\mathrm{T}$ cell activation. FEMS Immunol Med Microbiol 51, 535-546

46. Evrard B, Coudeyras S, Dosgilbert A, et al. (2011) Dosedependent immunomodulation of human dendritic cells by the probiotic Lactobacillus rhamnosus Lcr35. PLoS One 18, e18735.

47. Braat H, van den Brande J, van Tol E, et al. (2004) Lactobacillus rhamnosus induces peripheral hyporesponsiveness in stimulated $\mathrm{CD}^{+}{ }^{+} \mathrm{T}$ cells via modulation of dendritic cell function. Am J Clin Nutr 80, 1618-1625. 
48. D'Arienzo R, Maurano F, Lavermicocca P, et al. (2009) Modulation of the immune response by probiotic strains in a mouse model of gluten sensitivity. Cytokine 48, 254-259.

49. Foligne B, Zoumpopoulou G, Dewulf J, et al. (2007) A key role of dendritic cells in probiotic functionality. PLoS One 2, e313.

50. Mohamadzadeh M, Olson S, Kalina WV, et al. (2005) Lactobacilli activate human dendritic cells that skew $\mathrm{T}$ cells toward T helper 1 polarization. Proc Natl Acad Sci U S A 102, 2880-2885.

51. Weiss G, Christensen HR, Zeuthen LH, et al. (2011) Lactobacilli and bifidobacteria induce differential interferon- $\beta$ profiles in dendritic cells. Cytokine 56, 520-530.

52. Drago L, Nicola L, Iemoli E, et al. (2010) Strain-dependent release of cytokines modulated by Lactobacillus salivarius human isolates in an in vitro model. BMC Res Notes 3, 44.

53. Ivec M, Botić T, Koren S, et al. (2007) Interactions of macrophages with probiotic bacteria lead to increased antiviral response against vesicular stomatitis virus. Antiviral Res $\mathbf{7 5}$, 266-274.

54. Abbott A (2003) Biology's new dimension. Nature 424, $870-872$.

55. Schemeichel KL \& Bissell MJ (2003) Modeling tissue-specific signaling and organ function in three dimensions. $J$ Cell Sci 116, 2377-2388.

56. Zhang S (2004) Beyond the Petri dish. Nat Biotechnol 22, $151-152$.

57. Nickerson CA, Richter EG \& Ott CM (2007) Studying host-pathogen interactions in 3-D: organotypic models for infectious disease and drug development. J Neuroimmune Pharmacol 2, 26-31.

58. Bareiss PM, Metzger M, Sohn K, et al. (2008) Organotypical tissue cultures from adult murine colon as an in vitro model of intestinal mucosa. Histochem Cell Biol 129, 795-804.

59. Höner zu Bentrup K, Ramamurthy R, Ott CM, et al. (2006) Three-dimensional organotypic models of human colonic epithelium to study the early stages of enteric salmonellosis. Microbes Infect 8, 1813-1825.

60. Juuti-Uusitalo K, Klunder LJ, Sjollema KA, et al. (2011) Differential effects of TNF (TNFSF2) and IFN- $\gamma$ on intestinal epithelial cell morphogenesis and barrier function in three-dimensional culture. PLoS One 6, e22967.

61. Mappley LJ, Tchórzewska MA, Cooley WA, et al. (2011) Lactobacilli antagonize the growth, motility, and adherence of Brachyspira pilosicoli: a potential intervention against avian intestinal spirochetosis. Appl Environ Microbiol 77, 5402-5411.

62. Carol M, Borruel N, Antolin M, et al. (2006) Modulation of apoptosis in intestinal lymphocytes by a probiotic bacteria in Crohn's disease. J Leukoc Biol 79, 917-922.

63. Borruel N, Carol M, Casellas F, et al. (2002) Increased mucosal tumour necrosis factor alpha production in Crohn's disease can be downregulated ex vivo by probiotic bacteria. Gut 51, 659-664.

64. Borruel N, Casellas F, Antolín M, et al. (2003) Effects of nonpathogenic bacteria on cytokine secretion by human intestinal mucosa. Am J Gastroenterol 98, 865-870.

65. Mencarelli A, Distrutti E, Renga B, et al. (2011) Probiotics modulate intestinal expression of nuclear receptor and provide counter-regulatory signals to inflammation-driven adipose tissue activation. PLoS One 6, e22978.

66. Yan F, Cao H, Cover TL, et al. (2007) Soluble proteins produced by probiotic bacteria regulate intestinal epithelial cell survival and growth. Gastroenterology 132, 562-575.
67. Schanz J, Pusch J, Hansmann J, et al. (2010) Vascularised human tissue models: a new approach for the refinement of biomedical research. J Biotechnol 148, 56-63.

68. Unsworth BR \& Lelkes PI (1998) Growing tissues in microgravity. Nat Med 4, 901-907.

69. Nickerson CA, Ott CM, Wilson JW, et al. (2004) Microbial responses to microgravity and other low-shear environments. Microbiol Mol Biol Rev 68, 345-361.

70. Nickerson CA, Goodwin TJ, Terlonge J, et al. (2001) Threedimensional tissue assemblies: novel models for the study of Salmonella pathogenesis. Infect Immun 69, 7106-7120.

71. Carterson AJ, Höner zu Bentrup K, Ott CM, et al. (2005) A549 lung epithelial cells grown as three-dimensional aggregates: alternative tissue culture model for Pseudomonas aeruginosa pathogenesis. Infect Immun 73, 1129-1140.

72. Crabbé A, Sarker SF, Van Houdt R, et al. (2011) Alveolar epithelium protects macrophages from quorum sensinginduced cytotoxicity in a three-dimensional co-culture model. Cell Microbiol 13, 469-481.

73. LaMarca HL, Ott CM, Höner Zu Bentrup K, et al. (2005) Three-dimensional growth of extravillous cytotrophoblasts promotes differentiation and invasion. Placenta 26, 709-720.

74. Sainz B Jr, TenCate V \& Uprichard SL (2009) Three-dimensional Huh7 cell culture system for the study of Hepatitis C virus infection. Virol J 6, 103.

75. Howell JC \& Wells JM (2011) Generating intestinal tissue from stem cells: potential for research and therapy. Regen Med 6, 743-755.

76. Spence JR, Mayhew CN, Rankin SA, et al. (2011) Directed differentiation of human pluripotent stem cells into intestinal tissue in vitro. Nature 470, 105-109.

77. Cai J, Zhao Y, Liu Y, et al. (2007) Directed differentiation of human embryonic stem cells into functional hepatic cells. Hepatology 45, 1229-1239.

78. D'Amour KA, Agulnick AD, Eliazer S, et al. (2005) Efficient differentiation of human embryonic stem cells to definitive endoderm. Nat Biotechnol 23, 1534-1541.

79. Song Z, Cai J, Liu Y, et al. (2009) Efficient generation of hepatocyte-like cells from human induced pluripotent stem cells. Cell Res 19, 1233-1242.

80. Zhang D, Jiang W, Liu M, et al. (2009) Highly efficient differentiation of human ES cells and iPS cells into mature pancreatic insulin-producing cells. Cell Res 19, 429-438.

81. Basma H, Soto-Gutiérrez A, Yannam GR, et al. (2009) Differentiation and transplantation of human embryonic stem cellderived hepatocytes. Gastroenterology 136, 990-999.

82. Touboul T, Hannan NR, Corbineau S, et al. (2009) Generation of functional hepatocytes from human embryonic stem cells under chemically defined conditions that recapitulate liver development. Hepatology 51, 1754-1765.

83. Kroon E, Martinson LA, Kadoya K, et al. (2008) Pancreatic endoderm derived from human embryonic stem cells generates glucose responsive insulin-secreting cells in vivo. Nat Biotechnol 26, 443-452.

84. Koo BK, Stange DE, Sato T, et al. (2011) Controlled gene expression in primary Lgr5 organoid cultures. Nat Methods 9, 81-83.

85. McCracken KW, Howell JC, Wells JM, et al. (2011) Generating human intestinal tissue from pluripotent stem cells in vitro. Nat Protoc 6, 1920-1928.

86. Sato T, Stange DE, Ferrante M, et al. (2011) Long-term expansion of epithelial organoids from human colon, adenoma, adenocarcinoma, and Barrett's epithelium. Gastroenterology 141, 1762-1172. 